\title{
UTRICULARIA HUMBOLDTII SEED LONGEVITY
}

BARRY RICE •ELIZABETH M. SALVIA •P.O. Box $72741 •$ Davis, CA $95617 •$ USA •barry@sarracenia.com

Keywords: cultivation: seed treatment, U tricularia humboldtii.

The bladderwort U tricularia humboldtii Schomb. is an extraordinary plant in an extraordinary genus. It is a plant both well known and greatly desired by carnivorous plant horticul turists because of its enormous leaves and spectacular flowers; some growers even value it for its especially large bladders. Those interested in the natural history of carnivorous plants delight in its frequent occurrence within the water-filled urns of large bromeliads, including Brocchinia reducta, which may itself be carnivorous (Rice 2006).

As if ensuring that no aspect of it bores, $U$ tricularia humboldtii even has amazing seeds! They are large in outl ine (approximately $2-5 \mathrm{~mm}$ long and $1.5-2 \mathrm{~mm}$ wide) yet membranously thin and transparent. The middle portion of each seed bulges outwards, evoking the shape of a stuffed ravioli. In this case, the stuffing is a tiny, octopoid plantlet, al ready bright green and visible through the transparent seed walls. These seeds germinate immediately upon being moistened, like toy store compressed sponges that expand into brightly colored dinosaur and barnyard animals.

We were overjoyed when the U tricularia humboldtii we grow at the University of California (Davis) Botanical Conservatory flowered. Its flowers were enormous and flamboyant beyond expectations. And when selfed flowers produced seed, we enjoyed the spectacle of their rapid germination.

We took advantage of this event to investigate seed longevity, and the best way to prol ong their viability. As far as we knew, this avenue of study had not been explored. We al so made a few observations on the very early development of the seedlings. Finally, not wanting to be alone in our pleasure, we sent the bulk of our seeds to Travis Wyman, who selflessly di stributed seeds to all who contacted him

\section{Methods}

At maturity, the seed capsules of U tricularia humboldtii are approximately $1.5 \mathrm{~cm}$ long and resemble small pal e green olives in both shape and color. The membranous seed escape from the capsules by a ventral slit, presumably to be dispersed by wind. Our first fruit, from which we harvested approximately 500 seed for this experiment, matured on 28 March 2008.

We first tested the viability of twenty seeds by dropping them onto the surface of purified water in a Petri dish. The dish was placed $5 \mathrm{~cm}$ underneath six cool whitefluorescent bulbs with a $16 \mathrm{~h}: 8 \mathrm{~h}$ light:dark photoperiod. Daytime temperatures peaked at $31^{\circ} \mathrm{C}$, nighttime temperatures dropped to $24^{\circ} \mathrm{C}$. Within 24 hours, 18 of the seed had germinated. Reexamined after three and six days, no further seeds germinated.

We divided our ungerminated seed into three subsamples, stored in dry, loosely sealed Petri dishes (see Table 1). One subsample ("Room conditions") was stored in the dark at room temperatures. A second subsample ("Terrariumconditions") was placed in a 240-liter carnivorous plant 


\begin{tabular}{|l|c|c|c|}
\hline \multicolumn{4}{|l|}{ Table 1. Storage conditions for the three subsamples of U. humboldtii seed. } \\
\hline Subsample & Illumination & Temperature & $\mathrm{RH}$ \\
\hline Room conditions & $0: 24 \mathrm{~h}$ Light:Dark & $22 \pm 7^{\circ} \mathrm{C}$ & $47 \%$ \\
\hline Terrarium conditions & $17: 7 \mathrm{~h}$ Light:Dark & $23 \pm 7^{\circ} \mathrm{C}$ & $73 \%$ \\
\hline Refrigerated & $0: 24 \mathrm{~h}$ Light:Dark & $4 \pm 7^{\circ} \mathrm{C}$ & $25 \%$ \\
\hline
\end{tabular}

terrarium, approximately $40 \mathrm{~cm}$ beneath four fluorescent bul bs. This terrarium housed a collection of Pinguicula, U tricularia, young Nepenthes and seedling Sarracenia, and so was demonstrably an environment suitable for growing carnivorous plants. A third subsample ("Refrigerated") was placed in a refrigerator. The temperature and humidity in these three storage areas were measured using a digital infrared temperature scanner (Omega Engineering, OS90 series) and a digital sling psychrometer (Mannix, SAM 990DW).

Every three days, fifteen seeds from each subsample were dropped into Petri dishes filled with purified water. The dishes were placed $5 \mathrm{~cm}$ beneath a set of fluorescent bul bs. The dishes were inspected after one, three, and six days, and the numbers of seeds germinated were counted. We defined germination to have occurred if at least one of the arms of the seedling had emerged from the confines of the seed walls.

Results and discussion

Figure 1 shows the results for the seeds stored in the dark, at room temperature. The three sol id

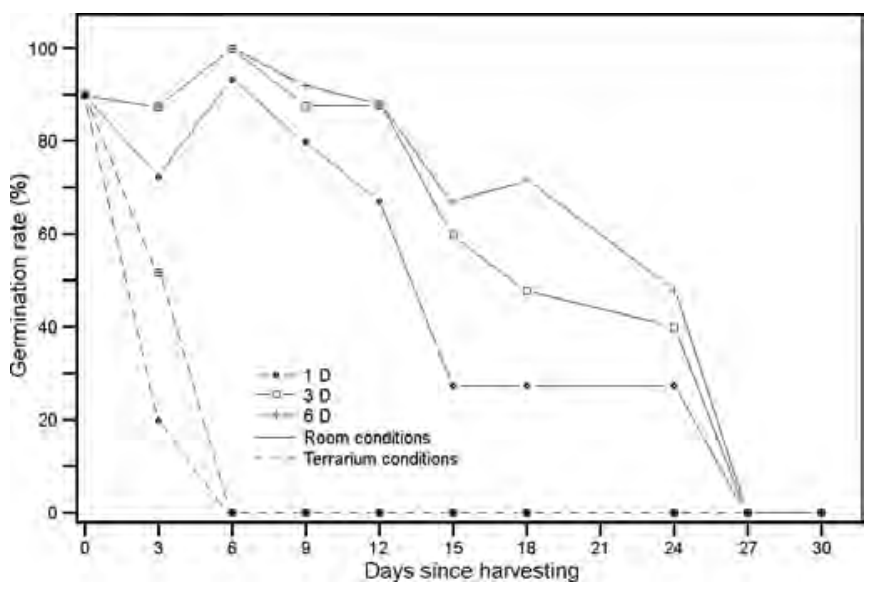

Figure 1: Germination rates for seeds, as measured 1 day, 3 days, and 6 days after being put in water. The $x$-axis indicates the seed age (number of days after capsule dehiscence) at which point the seeds were first moistened. The solid lines indicate results for seeds stored in the dark, at room conditions. Note the viability drops significantly after approximately 12 days. The dashed lines indicates results for seeds stored in an illuminated, humid terrarium; the viability plummets and the seeds are dead within just a few days. lines trace the germination rates measured 1 day, 3 days, and 6 days after being placed in water. Final germination rates are high, $80-100 \%$, for the first 12 days of storage. After that, seed viability drops steadily, reaching $0 \%$ viability at an age of approximately 27 days.

The data for the seeds stored inside a humid carnivorous plant terrarium are also plotted on Figure 1, but with dashed lines. Although this sample was stored in what might seem to be hospitable conditions, the setting was deadly for the seeds. After only 3 days the seed viability dropped to approximately $50 \%$, and after 6 days the seeds were 


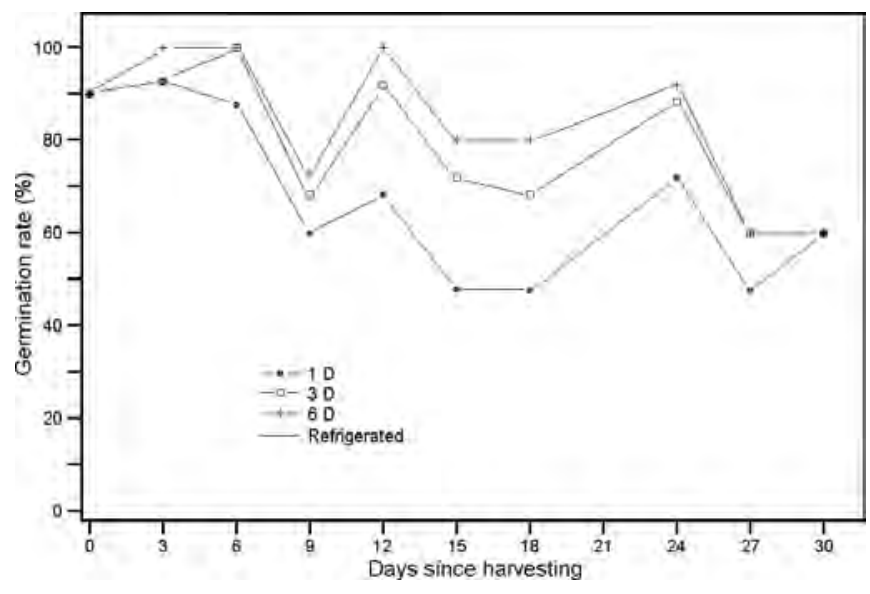

Figure 2: Germination rates for seeds stored in a refrigerator. The data presentation is as in Figure 1. Notice the seeds stay highly viable for at least a month. entirely nonviable Although the conditions were humid, it is likely that the high light levels forced the seedl ings in the transparent seeds to photosynthesize, and in the process the seeds dehydrated.

Interestingly, the seeds in cold storage survived the longest (see Figure 2). Germination rates exceeded $80 \%$ after 24 days. Even at 30 days-when the supply of seeds ran out for this experiment-germination rates were still at $60 \%$. While this result may not surprise managers

of seed banks, it is still interesting that col d storage was the best environment for these extremely delicate seeds of tropical species.

One would expect that in the wild, seeds are likely to be exposed to high light levels, and Figure 1 indicates that such seeds will have a very short lifespan. Finding a suitable environment to encourage germination must happen very quickly if the seeds are to survive.

Meanwhile, horticulturists would do best to harvest seeds of these plants immediately upon their rel easefrom the capsules. Even a few days of desiccation in normal growing conditions will decrease seed viability. The best way to store seeds is in the refrigerator or, barring that option, in the dark.

\section{Seedling observations}

Watching the seeds germinate over a matter of hours was fascinating. Seeing the seedlings almost crawl out of the seed coats, it was easy to forget they were not ani mals!

First, it became clear that the rapid germination is not due to a simple matter of the seed coat dissolving, either entirely or al ong fission lines. If such were the case, we would have seen the emergence of seedl ings even from dead seeds. Instead, germination appears to be a growth process caused by the extension of the curved, tentacular seedling arms. This process forces the seed coat to rupture (se Figure 3).

Upon emergence, the seedling consists of 6-8 filamentary, radially arranged leaves. Each leaf is ternately divided into three uniformly diverging arms. The leaves and its segments are strongly diverging, effectively filling a spherical volume (seeFigure 4).

A few days later, the seedling produces a new filamentary shoot from the center of the plantlet. This shoot elongates, and develops bladders and leaves. These early leaves are highly flattened. As the plant continues to grow, it devel ops into the familiar juvenile form consisting of many filmy, aquatic, divided leaves. After several months of good conditions, when the plant has gained sufficient biomass, it will produce the familiar paddle-shaped, aerial leaves. 


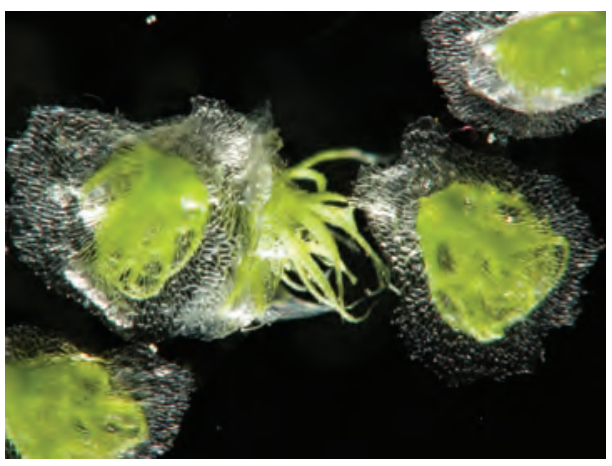

Figure 3: Utricularia humboldtii embryos can be observed while still within the seeds. The middle seed has just burst from its seed coat, and the seedling arms are still mostly recurved.

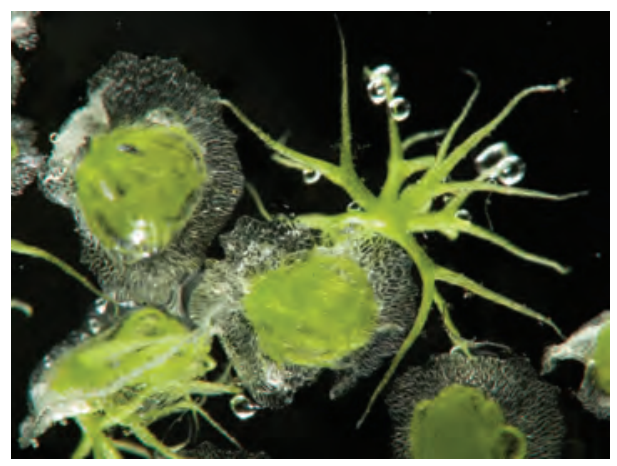

Figure 4: A slightly more advanced Utricularia humboldtii seedling, with its filamentary arms fully extended. Another seed is germinating at lower left.

Acknowledgements: We thank Tim Metcalf and Ernesto Sandoval at the University of California Davis Botanical Conservatory for their tireless efforts at growing fine plants such as U tricularia humboldtii.

\section{References}

Rice, B. 2006. Growing Carnivorous Plants. Timber Press.

Taylor, P. 1989. The Genus U tricularia: A Taxonomic Monograph. Kew Bulletin, Additional Series, XIV.

\section{THE ICPS SEED BANK \\ an exclusive menber benefil}

The International Carnivorous Plant Society offers its members exclusive access to a variety of carnivorous plant seeds. Seeds are ordered online at the ICPS Store:

\section{http://icps.clubexpress.com/}

The Seed Bank cannot exist without seed donations. Information about growing carnivorous plants from seed and donating seeds to the Seed Bank are at the ICPS public web site:

\section{http://www.carnivorousplants.org/seedbank}

If you do not bave access to the Internet, please send seed order form requests to:

International Carnivorous Plant Society

1564-A Fitzgerald Drive, PMB 322

Pinole, CA 94564-2229

JOHN BRITTNACHER, Seed Bank Manager, john@earnivorousplants.org 